\title{
ALAIN GIAMI
}

\section{De Kinsey au sida : l'évolution de la construction du comportement sexuel dans les enquêtes quantitatives.}

\author{
Sciences Sociales et Sante, 1991, vol IX, n 4, pp. 23-56.
}

Les recherches dans le champ de la sexualité connaissent depuis peu un renouveau et une impulsion que l'on peut mesurer au volume croissant des publications récentes à travers le monde (Gagnon: 1988; Turner: 1989, Tastemain: 1989). A la lecture de leurs cadres conceptuels et méthodologiques, nous avons remarqué une prédominance très nette des approches construites en termes de comportements sexuels et plus particulièrement de "comportements sexuels qui transmettent le VIH " (Turner: 1989). II importe donc de s'interroger sur le sens de I'utilisation de ce concept et ses implications par rapport aux stratégies de recherche et de construction d'objet, dans le contexte des approches de la sexualité en sciences sociales. Quels sont les points de vue privilégiés par le choix de ce concept ? Quels en sont les limites et les points aveugles?

Pour tenter de répondre à ces questions, nous avons repris quelques éléments de l'histoire du concept de comportement sexuel et de ses modes d'opérationnalisation dans quelques recherches fondées sur son utilisation. En effet, ce concept qui s'impose actuellement dans de nombreuses recherches sur la sexualité et qui opère ainsi un retour en force, est le produit d'une histoire et de débats qui n'ont pas été souvent clarifiés sur le plan théorique. Les recherches sur le comportement sexuel bénéficient d'un statut marginal dans les sciences sociales et d'un éparpillement qui a rendu difficile la production d'un savoir cumulatif et qui témoignent de ruptures souvent imposées par la conjoncture sociale. En outre, elles illustrent la mise en oeuvre d'une pluridisciplinarité au statut flou qui rend difficile le repérage de leur appartenance à un champ scientifique spécifique. Le concept de comportement sexuel a son origine dans les champs de la biologie et de la physiologie. Son exportation et son adoption dans le champ des sciences sociales ne peut être que problématique. "L'intérêt $d u$ sociologue pour la sexualité est évidemment différent de celui du biologiste. Le premier s'intéresse à l'incidence de ce comportement. Pour sa part, le sociologue veut découvrir des relations. En d'autres mots, il n'est pas intéressé fondamentalement par des nombres ni par des courbes de distribution, mais par ce que le comportement signifie en termes d'attitudes de la société et de la personne elle-même. (...) Pour le sociologue, le phénomène biologique 
ne constitue pas le centre d'intérêt du comportement sexuel. " (Burgess: 1949, p.228)1. La focalisation sur les comportements sexuels nécessite donc, d'une part, l'élucidation des conditions d'utilisation d'un concept issu de la biologie dans le champ des sciences sociales et d'autre part, le repérage des problématiques sociologiques et psychologiques mises en oeuvre explicitement ou implicitement pour expliquer et comprendre ce qui est désigné comme comportement, dans un autre registre que celui de la biologie.

\section{LE COMPORTEMENT SEXUEL : Frontières et limites d'un concept}

Le concept de "comportement sexuel" résulte de l'association entre le concept de "comportement" qui définit un type et une modalité d'activité humaine et le qualificatif de "sexuel" qui en définit le champ. II est nécessaire d'examiner la portée et les limites de cette association entre un concept qui a un sens extrêmement précis et un qualificatif qui semble être toujours resté enrobé de polysémie.

Lalande définit le comportement comme suit: "Le comportement d'un être est l'ensemble des réactions globales de son organisme, tant communes à l'espèce que particulières à l'individu." (Lalande, 1962: p.156). Lagache le présente et le discute dans son cours sur la conduite humaine: "Le comportement est l'étude des mouvements extérieurs ou extérieurement visibles, ce n'est pas la conscience " ; "Chez les behavioristes orthodoxes c'est la psychologie de la secousse musculaire "; "Guillaume a défini le comportement comme se réduisant à des faits matériels. Nous sommes là en présence d'une réduction physique et physiologique. Le behaviorisme est une position doctrinale dans laquelle la réduction physique est un postulat. (....); à l'origine, behavior, c'est ce qui est accessible à l'observateur." (Lagache: 1948, p.5).

Ce concept est caractérisé par l'extériorité et l'observabilité qui contribuent à une spécification minimale de l'activité humaine ainsi qu'à la réduction des explications et des significations qu'elle pourrait revêtir. II renvoie à un ensemble de réactions de l'organisme dont "la conscience" est exclue, y compris en tant que réaction de l'organisme.

II faut donc s'interroger sur la spécification, la réduction et l'extension qu'impose au champ du sexuel son association avec le concept de comportement.

Dans l'Encyclopaedia Universalis, l'entrée "comportement sexuel" est principalement consacrée au comportement sexuel animal2:

${ }^{1}$ Les citations américaines ont été traduites par l'auteur (A.G.).

2 par contre l'entrée "sexualité" traite de la sexualité humaine des points de vue phylogénétique, psychanalytique et sociologique. 
"à partir d'un signal simple et bien défini, à savoir la modification du taux de stéroïdes sexuels dans la circulation sanguine, on voit se produire une modification radicale des relations interindividuelles." (Signoret: 1980, p. 934).

Malgré sa détermination endocrinologique le comportement sexuel animal est replacé dans "l'ensemble du contexte social de l'espèce" sur lequel il produirait des modifications. Sur ce premier fondement, le comportement sexuel humain est défini de manière beaucoup plus complexe:

"Le comportement sexuel humain apparaît comme le terme d'une évolution où la part prise par le système nerveux devient dominante, tandis que le système hormonal, tout en restant présent et actif, perd de son importance pour n'être que facultatif."(....) On tendrait à considérer l'ensemble du domaine sexuel comme ressortissant à la biologie, en limitant la dimension socio-culturelle à l'addition d'un mélange de permissions et d'interdits à un phénomène purement physiologique. Or il semble possible, au contraire, de discerner en de nombreux domaines une participation extrêmement importante et souvent dominante des influences socio-culturelles, souvent là où on les attendrait le moins." (Signoret: 1980, p. 934).

Cette deuxième définition procède d'un renversement de perspective dans lequel les dimensions socio-culturelles auraient un rôle sur l'élaboration et l'expression du comportement sexuel.

Gebhard, un des associés de Kinsey, donne sa définition du comportement

sexuel après en avoir exclu les aspects liés au sexe (genre) et à la reproduction:

"Le comportement sexuel peut être défini de la meilleure façon comme ces mouvements, vocalisations et réactions directement impliqués avec l'émergence des réponses physiologiques qui constituent en partie, l'excitation sexuelle et qui lorsqu'elles sont poursuivies - devraient ordinairement aboutir à l'orgasme." (Gebhard: 1971, p.251).

"Le comportement sexuel peut être défini comme toute activité - solitaire, entre deux personnes, ou en groupe - qui amène de l'excitation sexuelle." (Gebhard: 1974, p.593).

Enfin, le sexologue G. Zwang replace de manière radicale le comportement sexuel dans le champ de la biologie et de la nature animale de l'homme:

"Longtemps hostile à son animalité autant qu'à ses déterminismes instinctifs, l'humanité dut reconnaître l'existence imprimée dans ses gènes, de schémas comportementaux obligatoires, inhérents à sa constitution biologique. S'inscrivant dans le cadre d'une biologie comportementale, la science des comportements, l'éthologie proposa donc aux humains de mieux se connaître pour "se" mieux vivre. (...) La sexologie couvre donc un domaine étendu de la biologie, et c'est ainsi qu'elle doit s'inscrire dans la somme des connaissances scientifiques humaines. Donnant une vue globale du comportement sexuel des hommes et des femmes, autant que de son fondement anatomique et physiologique, et de ses retentissements culturels sociaux, moraux, etc..." (Zwang: 1990, p.4).

Les définitions du comportement sexuel ne sont pas unanimes. Le débat oppose deux conceptions:

1/. l'assimilation et la réduction de l'ensemble des activités et des réactions définies comme sexuelles, au comportement sexuel, c'est-à-dire résultant d'une 
excitation définie elle-même comme sexuelle, sans plus de spécification, et constituant le substrat physiologique. Ce terme n'est retenu que pour décrire une classe d'actes qualifiés de manière générique et non pas pour décrire des types précis d'actes dans leur singularité.

2/. la prise en compte avec l'activité, d'un certain nombre de dimensions, réductibles ou non au substrat physiologique, et pouvant aussi être considérées comme des modifications d'état associées au sexuel et impliquant les dimensions psychiques, culturelles et sociales. Le comportement sexuel apparaît alors comme la résultante d'un ensemble de facteurs diversement intriqués.

Dans les deux cas, le comportement sexuel n'est pas défini du point de vue de son extension ni de ses limites concernant des actes spécifiques. II s'agit d'un concept générique. On remarque notamment que le comportement sexuel n'est pas défini en relation avec des zones corporelles spécifiques qui constitueraient le lieu de son expression, et qu'il n'est pas non plus placé "sous le primat de la reproduction". Plus fondamentale pour notre propos, la question de l'intrication ou de l'articulation des différentes dimensions constitutives du sexuel ne fait pas l'objet d'un consensus.

II s'agit donc de comprendre, d'une part, la place du comportement sexuel dans l'ensemble désigné comme sexualité et, d'autre part, son statut par rapport aux champs psychologique et sociologique.

\section{METHODE}

Le "comportement sexuel" fonctionne comme un paradigme scientifique (Kuhn: 1970) dans la mesure où il constitue une communauté de consensus entre des recherches qui n'interrogent pas son statut théorique et qui produisent à partir de son utilisation et de son opérationalisation un certain nombre de constructions interprétatives.

Le paradigme comprend:

* Les concepts théoriques énoncés, ou pas, dans les recherches et les définitions opérationnelles qui participent de la construction des problématiques;

* Les instruments de recueil d'informations et notamment la structure et les contenus des questionnaires utilisés. ${ }^{3}$

* Les modèles, classifications, typologies, échelles etc... construits pour exprimer les synthèses des résultats.

${ }^{3} \|$ serait intéressant d'analyser les modes de passation de ces questionnaires comme des scénarios interpersonnels qui se manifestent par l'ordre d'apparition des questions (structuré ou semi-directif), l'organisation des différentes parties en "face à face", en "auto administré", par téléphone, par voie de presse, etc...) et qui permettent de repérer les modalités socialement acceptables d'une interlocution sur la sexualité. 
Notre corpus est constitué de recherches qui visent à un recensement de l'activité sexuelle de la population générale de manière à produire une représentation globale de cette activité et d'en expliquer le fonctionnement et les variations, voire d'en repérer les modifications. II s'agit des Rapports Kinsey $(1948,1953)$, et du Rapport Simon (1972) pour ce qui concerne les recherches réalisées et publiées avant l'apparition du sida 4 . Les recherches menées depuis l'apparition du sida en sont à des stades d'avancement variables. Nous avons pris en compte, les projets de recherche (Action Concertée de la Communauté Européenne A.C.C.E. : 1989; NORC 5 : Laumann, Gagnon, Michael : 1989 ; OMS : c. 1989) auxquels nous avons pu avoir accès ${ }^{6}$. Notre analyse comparative de l'ensemble de ces recherches prend appui sur les définitions conceptuelles du "comportement sexuel", sur les structures et les contenus des questionnaires utilisés, et sur les typologies construites dans les recherches publiées. Nous n'avons pas travaillé sur les méthodes de traitement statistique ni sur les résultats quantitatifs. Les limites de notre compétence ne nous permettent pas d'évaluer leur pertinence ou leur rigueur. Enfin nous n'avons pas pris en compte les nombreuses enquêtes réalisées et publiées dans la presse au cours des années 70 et qui peuvent faire l'objet d'un traitement spécifique.

Ces recherches sont significatives du fait de leur petit nombre, de l'importance des moyens dont elles ont disposé, et de l'événement culturel qu'elles constituent. Elles représentent, pour ces raisons, des modèles de référence difficilement contournables. Malgré cela, elles sont souvent considérées comme non-comparables et non-cumulatives du fait de la différence de leur mode d'échantillonnage et de la structure de leur questionnaire.

Nous voulons démontrer que les difficultés de comparaison et de cumulativité de ces recherches sont davantage liées à la contingence de leurs problématiques. En dehors du Rapport Kinsey qui répond à une nécessité sociale difficilement analysable (la mythologie qui entoure ce travail a pour effet d'obscurcir ses conditions sociales de production; la "personnalité" de Kinsey et son "acharnement au travail" sont les explications les plus communément avancées), ces recherches répondent à une forte demande sociale qui a pour effet de reconstruire et de mettre en oeuvre des définitions du concept qui sont profondément déterminées par les préoccupations du

\footnotetext{
${ }^{4}$ Nous désignons par "sida" la dimension globale du phénomène et notamment sa dimensions culturelle, et par "VIH" sa dimension strictement bio-médicale.

${ }^{5}$ National Opinion Research Center, Université de Chicago.

6 La problématique du présent article a été élaborée à partir des questions qui sont apparues dans le cadre de notre participation à l'ACSF (Analyse des Comportements Sexuels des personnes résidant en France). Nous n'avons pas pris en compte cette recherche pour des raisons de déontologie scientifique.
} 
moment. II s'agit notamment de comprendre l'influence qu'ont exercé les thèmes de la contraception et de la maîtrise de la procréation (Simon) et le rôle que joue actuellement le thème du sida, dans la construction des objets de recherche. L'analyse de ces recherches nous permet de montrer comment ces préoccupations ont constitué des biais pour la construction de l'objet "comportement sexuel" en mettant l'accent sur certaines de ses composantes et en en abandonnant d'autres. II s'agit donc de montrer que des recherches qui prétendent traiter de "l'ensemble de l'activité sexuelle" ne traitent effectivement que d'une partie plus ou moins importante de celleci et que les éléments retenus sont censés constituer l'ensemble. Ces processus de sélection et les résultats auxquels ils aboutissent peuvent être interprétés comme l'expression de constructions sociales du comportement sexuel.

La démarche comparative est la seule démarche permettant de considérer ces travaux comme des constructions et d'identifier leurs points de focalisation et leurs lacunes respectifs. II serait tout à fait illusoire de croire que l'on puisse procéder à un "inventaire exhaustif" de l'activité sexuelle (Gaignebet: 1991, p.881) pouvant servir d'étalon de mesure, tant la diversité des pratiques et des significations qui les accompagnent et les structurent est immense. Le découpage et la sélection ne peuvent être que la règle en la matière. Encore faut-il se doter de critères rigoureux et cohérents. C'est la comparaison entre les structures du comportement sexuel construites par Kinsey et par Simon, qui nous permettra de considérer les définitions implicites et explicites de celles qui sont construites actuellement comme de nouvelles constructions orientées par le sida.

Partant de l'hypothèse selon laquelle les contextes culturels et la demande sociale ont produit des effets de sélection et de reconstruction du champ du sexuel, nous proposons donc de comprendre comment le contexte du sida oriente la construction des objets et des problématiques dans les recherches menées actuellement dans le champ de la sexualité. Etant entendu que le choix du concept de comportement sexuel pour traiter de ces problèmes est bien évidemment l'un des effets du contexte. Récemment, d'autres travaux ont traité de la construction sociale de la sexualité en se fondant sur la démarche historique (Laqueur: 1990) ou bien sur une démarche anthropologique (Caplan: 1987).

\section{KINSEY: la mesure des orgasmes}

L'étude du comportement sexuel de l'espèce humaine apparaît de manière systématique avec Alfred Kinsey. C'est-à-dire que pour la première fois, on fait le projet explicite et l'on met en œuvre l'ambition de cerner la totalité de l'activité sexuelle de l'espèce humaine du point de vue de ses différents modes d'expression et ce, au moyen d'une investigation auprès d'un échantillon supposé représentatif de l'ensemble de la population américaine. Cette démarche constitue une rupture, d'une part, avec 
les grandes oeuvres de sexologie clinique de la fin du $X I X^{\circ}$ et du début du $X X^{\circ}$ siècle qui visaient à décrire certaines des composantes de l'activité sexuelle, en se focalisant sur les "déviations" (Krafft-Ebing: 1965, Ellis: 1936), d'autre part, avec la théorie freudienne de la sexualité, et, avec les modes d'appréciation de la sexualité fondées sur la morale religieuse, la médecine ou la criminologie (Trilling: 1948; Lanteri Laura : 1979). Kinsey fait entrer la sexualité dans le champ des sciences naturelles en tirant son argumentation de la zoologie, de la biologie, et de la physiologie et en ayant recours à la statistique et à des facteurs d'ordre sociologique. II se situe cependant dans le droit-fil de la modernité sexuelle fondée sur la reconnaissance du caractère positif de l'activité sexuelle (Robinson: 1976) dont il veut élaborer une connaissance essentiellement descriptive des pratiques effectives, dépourvue de toute connotation morale et de tout jugement a priori. II s'insère de plein pied dans ce que Foucault (1976) appelle la mise en discours de la sexualité en utilisant principalement la biographie sexuelle comme moyen de recueil des informations. Plus de douze mille personnes ont été longuement interrogées (et certaines plusieurs fois) par Kinsey et ses collègues (Pomeroy: 1972). L'instrument utilisé n'est pas standardisé; il comporte un tronc commun de 300 questions environ qui constitue le minimum à faire passer aux personnes qui ont un minimum d'activité sexuelle. L'échantillon est construit selon la méthode dite "boule de neige" en constituant des groupes à 100\%. C'est l'ensemble de certains sous-groupes sociaux de différentes caractéristiques qui est considéré comme représentatif de la population américaine. Ces groupes sont abordés à $100 \%$ de leurs membres.

L'oeuvre de Kinsey constitue le déploiement d'un vaste systême doté d'une certaine cohérence. II importe cependant d'identifier le sens et les limites de son propos en partant de l'étude de la définition opérationnelle qu'il donne du comportement sexuel. Cette limitation apparaît à la lecture de la comparaison entre les intentions et les définitions générales d'une part, le guide d'entretien d'autre part, et enfin la construction synthétique fondée sur une analyse quantitative, à l'aide de laquelle il présente ses résultats. II s'agit donc de repérer ce qui, pour Kinsey constitue le comportement sexuel, ce qui en est exclu ou occulté tout en pouvant être considéré comme relevant du sexuel.

\footnotetext{
"Notre étude tient compte de toutes sortes d'individus et de tous les aspects du comportement sexuel. Les cas étudiés, ainsi que les résultats obtenus sur chaque cas, ont été choisis sans aucune notion préconçue de rareté ou de banalité, de normal ou d'anormal, de valeur morale ou sociale. Une telle discrimination aurait empêché d'établir les faits de façon précise. L'étude impartiale du comportement sexuel a été considérablement entravée par le fait que certains aspects de ce comportement sont presque universellement considérés - même dans les milieux scientifiques - comme normaux et d'autres comme anormaux. La similitude des distinctions faites entre les termes normaux et anormaux, et les termes bien et mal, démontre amplement les origines philosophiques, religieuses et culturelles de ces concepts. (....) Notre étude est avant tout une description de ce que font les hommes,
} 
et non de ce qu'ils devraient faire, ni des catégories d'hommes qui agissent ainsi. Elle décrit le comportement sexuel de l'américain tel que nous le rencontrons. (...) Nous avons fait l'analyse impartiale de tous les genres d'activité sexuelle qu'on trouve dans toutes les catégories d'hommes.(...) Notre étude embrasse tous les aspects du comportement sexuel humain, et non son aspect biologique, psychologique ou sociologique pris isolément. " (Kinsey: 1948, p. 25).

"Le comportement sexuel de l'homme est le résultat de son organisation morphologique et physiologique, ainsi que de la situation où l'ont mené ses expériences, jointes à l'ensemble des êtres et des choses qui l'entourent. (...) il est soumis à des facteurs biologiques, psychologiques et sociologiques, mais tous ces éléments agissent simultanément et à la fin on se trouve en présence d'un seul phénomène formant un tout qui n'est pas purement biologique, psychologique ou sociologique dans son essence." (Kinsey: 1948, p.428).

On est frappé par l'ambition de totalité et d'exhaustivité que l'on découvre sous la plume de Kinsey et qui se déploie par rapport à un certain nombre de dimensions du phénomène. Le "comportement sexuel" décrit par Kinsey prend en compte des types d'activité sexuelles différents, indépendamment de leur rapport à la norme ou de leur fréquence et autant que faire se peut, la totalité de ces activités. II apparaît cependant que cette exhaustivité n'a pas été réalisée en ce qui concerne les aspects de l'activité sexuelle, et les différentes composantes de celle-ci.

La seule limite reconnue par Kinsey porte sur le nombre des personnes interrogées. Dans l'exergue du Rapport sur le comportement sexuel de l'homme ,Kinsey remercie d'avance "les quatre-vingt huit mille personnes qui, un jour, aideront à terminer cette étude" (!). II a été montré par ailleurs que cette totalité n'avait pas été réalisée sur le plan de l'échantillon qui témoigne d'une surreprésentation des individus appartenant à la classe moyenne blanche du Centre et du Nord-Est des Etats-Unis.

\section{le critère du sexuel: l'orgasme}

Avant de décrire l'étendue du champ des activités considérées comme sexuelles, il est nécessaire de rappeler le critère essentiel que Kinsey utilise pour spécifier une activité comme sexuelle: l'orgasme .

"Les calculs de fréquence contenus dans le présent ouvrage ne prennent en considération que les activités sexuelles ayant conduit à l'orgasme, quoique bien d'autres aspects du comportement sexuel humain doivent être envisagés." (Kinsey: 1948, p.160).

La définition est précisée et nuancée dans le volume consacré au comportement sexuel de la femme:

"L'orgasme est un phénomène distinct et particulier que l'on peut généralement reconnaître aussi facilement chez l'homme que chez la femme. Nous l'avons donc pris comme unité concrète pour déterminer à la fois les incidences et les fréquences. L'emploi d'une telle unité est justifié par le fait que l'orgasme qu'il provienne de la masturbation, du coït conjugal, des caresses ou de toute autre source permet au sujet de se libérer physiologiquement de son excitation sexuelle; cependant la signification sociale de l'orgasme peut varier selon le type d'activité qui l'a provoqué; aussi l'emploi de ce phénomène comme unité de mesure a-t-il soulevé quelques objections." (Kinsey: 1953, p.60). 
Concernant les hommes, Kinsey considère que l'éjaculation est la conséquence, et donc la preuve, de la réaction neuromusculaire que constitue l'orgasme et qu'il est "rare qu'il se livre à des activités comme la masturbation ou le coït sans les pousser jusqu'à l'orgasme " . La généralité supposée de l'occurence de l'orgasme chez les hommes conduit Kinsey à utiliser ce critère pour caractériser les activités considérées comme sexuelles.

Par contre, les objections majeures à l'emploi du critère de l'orgasme pour définir le caractère sexuel de ces activités apparaissent à propos de la définition du comportement sexuel des femmes et des enfants.

En ce qui concerne les femmes, Kinsey constate que de nombreuses femmes déclarent ne pas éprouver d'orgasme lors de leurs activités sexuelles. Kinsey, fidèle à son critère ne prendra en compte que les activités ayant abouti à l'orgasme. Ce qui constituera l'un des éléments lui permettant d'expliquer la fréquence inférieure d'activité sexuelle totale de la femme par rapport à celle de l'homme (Kinsey: 1953, p. 94). Kinsey est en outre amené à introduire une distinction entre satisfaction et orgasme pour expliquer la fréquence de certains types d'activités sexuelles (notamment le coït conjugal).

En ce qui concerne les enfants, Kinsey se fonde sur un nombre peu significatif (sur le plan statistique) d'enfants et d'adolescents pré-pubères qui ont expérimentés l'orgasme. Pour Kinsey, si l'éjaculation est la conséquence de l'orgasme, celui-ci peut se dérouler en l'absence d'éjaculation. II explique la difficulté de comptabiliser cette expérience par l'absence d'éjaculation et par le manque d'expérience qui rend difficile la prise de conscience qu'il s'agit bien d'un orgasme.

Malgré, les contradictions et les limitations qui témoignent d'une inadéquation relative de la notion d'orgasme pour désigner le sexuel, Kinsey maintient ce critère; ce qui lui permet d'inclure l'excitation, comme point de départ, "motivation", à s'engager dans un comportement sexuel. Cependant, il ne considére pas l'excitation comme critère suffisant pour définir le caractère sexuel d'une activité. Par contre, on peut penser qu'il n'a pas retenu dans son décompte toutes les activités, leurs aspects et les situations qui peuvent mener à l'orgasme et qu'il en a retenu certaines qui ne sont pas automatiquement associées à l'orgasme. Ce qui laisse à penser que d'autres critères ont été à l'oeuvre plus ou moins à l'insu de Kinsey.

\section{les aspects de l'activité sexuelle}

Kinsey entreprend la comptabilité de certains comportements définis comme sexuels, c'est à dire considérés comme moyens de parvenir à l'orgasme. II identifie les modalités (sources) d'obtention de la totalité des orgasmes ("exutoire sexuel total ") obtenus et le sexe (genre) des individus qui y sont associés, lorsque la modalité nécessite la présence d'un ou de plusieurs tiers (activités socio-sexuelles) . 
D'autre part, il explique les variations des quantités et des modalités d'obtention des orgasmes en fonction d'un certain nombre de facteurs biologiques (le sexe et l'âge,) et socio-culturels (la classe sociale, le niveau d'instruction, la religion). "Le Comportement Sexuel de l'Homme repose sur deux concepts fondamentaux: l'exutoire et le facteur. L'exutoire désigne toute activité qui se conclue par un orgasme alors que le facteur renvoie à toutes les circonstances non-sexuelles qui peuvent jouer un rôle sur le choix ou la fréquence de l'exutoire." (Robinson: 1976, p. 58).

Pour ce faire Kinsey met au point un guide d'entretien visant à recueillir les biographies sexuelles; il comporte neuf sections qui comportent elles-mêmes des sous-sections très détaillées qui prennent en compte les exutoires et les facteurs. 1: Données économiques et sociales, 2: Histoire de la vie conjugale (pour chaque mariage), 3: éducation sexuelle, 4: données physiques et physiologiques, 5: Rêves sexuels, 6: masturbation, 7: histoire hétérosexuelle, 8: histoire homosexuelle, 9: Contacts animaux.

Les items contenus dans le guide d'entretien permettent d'embrasser une étendue de pratiques, d'attitudes et de sentiments beaucoup plus vaste que ce qui sera retenu au moment de la présentation des résultats qui, pour sa part, témoignera d'une réduction de l'ensemble couvert par les biographies. Ne seront finalement retenus comme relevant du comportement sexuel que certains des actes (supposés) effectifs, rapportés par les individus interrogés. Les attitudes et réactions psychologiques viendront émailler les digressions nombreuses de Kinsey. II établit donc un continuum entre: la masturbation, les pollutions nocturnes, les caresses sexuelles, les coïts (pré-conjugaux, conjugaux, et extra-conjugaux), les activités homosexuelles et les rapports sexuels avec les animaux. (Kinsey: 1948, p.265).

Kinsey se montre soucieux de mettre en lumière la fréquence de conduites considérées comme mineures ou tabou telles que les rêves sexuels, la masturbation, les caresses hétérosexuelles pour montrer que cette fréquence est corrélée à celle des rapports hétérosexuels adultes. De même, il prend un malin plaisir à faire apparaître l'importance des rapports sexuels pré-conjugaux et des activités homosexuelles afin de contrer les idées reçues en la matière. Enfin, le choix de son mode de présentation relativise la centralité des rapports hétérosexuels dans le décompte de l'activité sexuelle totale. ${ }^{7}$

En outre, Kinsey situe les sources de l'orgasme par rapport à la dimension socio-sexuelle, c'est à dire en fonction du sexe (genre) du partenaire. Kinsey récuse les oppositions strictes entre homosexualité et hétérosexualité car elles ne tiendraient pas compte de la réalité des activités effectives. II s'oppose surtout à la "signification

\footnotetext{
7 Sur les conceptions idéologiques de Kinsey voir: W. Pomeroy op. cit.
} 
sociale" de l'homosexualité qui range dans cette catégorie tout individu ayant eu, ne serait-ce qu'une activité avec un partenaire du même sexe. II construit donc "l'échelle H.-H." (hétérosexualité - homosexualité) qui distribue à nouveau les individus sur un deuxième continuum en sept points. II situe aux deux extrêmes, les individus qui ont exclusivement des réactions et des activités orientées vers un ou des individus de sexe différent (les catégories 0 et 6 ); la catégorie 3 regroupe "les individus qui se situent à mi-chemin de l'hétérosexualité et de l'homosexualité. Ils sont à la fois homosexuels et hétérosexuels dans leur activité et - ou - par leurs réactions psychiques." Les catégories 1 et 2 regroupent les individus qui sont surtout hétérosexuels et qui ont soit des réactions psychiques, soit des activités sexuelles "accidentelles " avec des partenaires du même sexe; et les catégories 4 et 5 regroupent les individus qui ont surtout des activités homosexuelles et des réactions psychiques ou des activités "accidentelles " avec des personnes de sexe différent. Enfin la catégorie " $X$ " regroupe les individus qui n'ont aucune réaction psychique et aucune activité avec un partenaire. (Kinsey: 1948, p.807).

L'activité sexuelle totale d'un individu est donc comptabilisée sur deux axes: les sources et le nombre relatif de partenaires de l'un ou de l'autre sexe. Leur fréquence et le choix d'y avoir recours est expliqué par l'influence des facteurs sociaux et biologiques.

\section{les lacunes et les limites : le champ du sexuel et du non-sexuel}

La constatation et l'analyse des lacunes et des limites ne peut s'effectuer que sur la base d'une comparaison avec les éléments présents dans les autres recherches. En tout état de cause, la totalité ou l'exhaustivité ne peuvent qu'être, en la matière, asymptotiques.

Sur le plan des pratiques, il reste étonnamment flou et discret sur la description des techniques sexuelles employées par les individus et sur le décompte de leur fréquence. II se montre très avare en informations concernant les rapports buco-génitaux (cf. Gagnon, Simon: 1987) et les rapports anaux. ${ }^{8}$

Sur un autre versant, la procréation (contraception, avortement, grossesse) et les maladies vénériennes sont absents. Ainsi par exemple, la peur de la grossesse, le désir d'enfant ou la peur des maladies vénériennes ne sont pas pris en compte ni comme facteurs physiologiques, ni comme facteurs psychologiques pouvant être mis en relation avec les fréquences ou les choix d'activité sexuelle, ni chez l'homme ni chez la femme.

8 il faut cependant rappeler que dans de nombreux états américains, ces pratiques constituent des délits même lorsqu'elles se déroulent entre adultes consentants. 
Ces lacunes dévoilent en négatif la conception que Kinsey se faisait du champ du sexuel. Celui-ci est caractérisé par une configuration centrée sur le caractère effectif d'une activité, sa fréquence et sa représentativité au niveau statistique, et son articulation avec l'orgasme. La catégorie centrale de cette recherche réside dans la quantité. Ainsi, par exemple, le nombre d'orgasmes obtenus par les femmes avant leur accession à la conjugalité (masturbation, caresses manuelles, rapports préconjugaux, etc..) est en étroite corrélation avec le nombre d'orgasmes obtenu dans les rapports conjugaux. De même, à l'intérieur de la situation conjugale et pour une période donnée les nombres d'orgasmes pour chaque forme d'exutoire sont fortement corrélés. En d'autres termes, plus on obtient d'orgasmes d'une certaine façon plus on cherche à en obtenir par d'autres moyens.

"Plus de la moitié (50 à $57 \%$ ) des femmes ayant pratiqué un coït préconjugal érotiquement satisfaisant, obtinrent l'orgasme presque à chaque coït pendant leur première année de mariage. De celles n'ayant pas pratiqué le coït préconjugal et n'ayant obtenu l'orgasme par aucun moyen avant leur mariage, $29 \%$ seulement ont eu près de $100 \%$ d'orgasmes pendant leur première année de mariage." (Kinsey: 1953, p.314).

La quantité explique et produit la quantité et la quantité reste l'indicateur de la qualité (satisfaction). La catégorie de la quantité fonctionne dans le système de Kinsey comme "facteur" déterminant. A l'inverse, c'est le même critère qui est utilisé pour justifier l'absence de certaines activités dans le décompte de l'activité sexuelle totale.

La centration sur les actes exclut, en outre, la dimension de l'identité ou des dispositions psychologiques. Par exemple, Kinsey, qui a fait apparaître que $37 \%$ des hommes avaient eu au moins un contact de type homosexuel ayant abouti à l'orgasme, s'est toujours refusé à employer le terme "homosexuel" autrement que comme un adjectif servant à désigner les caractéristiques socio-sexuelles d'un type d'activité. En ce sens, il rompt avec les conceptions psycho-pathologiques et avec l'approche psychanalytique de la sexualité qui établissent un lien entre les pratiques et la subjectivité ou la structuration psychologique identitaire de l'acteur. Pour Kinsey, la fréquence et le choix de certaines activités par un individu ne permettent pas de déduire "qui il est" sur le plan psychologique mais de repérer les influences que le milieu a exercées sur lui en modelant son expérience. Pour Kinsey, les actes sexuels sont principalement déterminés par des causes biologiques et physiologiques et leur fréquence et leur choix dépend de facteurs non-sexuels tels que la classe sociale, le niveau d'éducation, la religion ainsi que du nombre d'orgasmes cumulés. Kinsey se plait à donner des exemples nombreux d'individus qui ont des fréquences et des types d'activité sexuelle "surprenantes" par rapport à leur statut ou leur insertion sociale. 
On est cependant frappé par l'absence de certains aspects et notamment des activités voyeuristes et exhibitionnistes, du fétichisme, et des activités de type sadomasochiste, dans la prise en compte de l'activité sexuelle totale. Les composantes voyeuristes et exhibitionnistes sont évoquées à propos des facteurs psychologiques des réactions sexuelles. Le sado-masochisme est évoqué à propos du rôle des "morsures" sur l'excitation sexuelle.L'absence des ces composantes de l'activité sexuelle dévoile la "qualité" naturaliste et positive que Kinsey attribue à la sexualité. D'une part, Kinsey n'accorde qu'une part restreinte dans son schéma explicatif aux scénarios et aux fantasmes sexuels ou non-sexuels au titre de composante du comportement sexuel. D'autre part, il en exclue les formes associées ou intriquées à l'agressivité. La discussion qui porte sur l'objet des rêves associés aux pollutions nocturnes est particulièrement révélatrice. Pour Kinsey, le rêve est principalement le reflet de l'expérience sexuelle réelle de l'individu, dans laquelle il trouve son ancrage et son matériel alors que "les rêves qui n'ont aucune corrélation avec l'expérience vécue (...) ne sont pas fréquents" (Kinsey: 1948, p. 664). Y compris au niveau de l'imaginaire, le sexuel n'est produit que par du sexuel et ne produit que du sexuel. Ce qui est cohérent avec le fait que Kinsey nie l'importance et même l'existence de la sublimation (au sens freudien). Le sexuel de Kinsey est explicitement un sexuel "sans qualité", situé quasiment en position d'extériorité et d'objectivité par rapport à l'individu et sans rapport de type qualitatif avec l'idéologie ou la personnalité de l'individu.

\section{SIMON: la sexualité contraceptée}

Le Rapport sur le comportement sexuel des Français publié en 1972 sous la direction de Pierre Simon reste, à notre connaissance, le seul document comparable (toutes proportions gardées), sur le plan de son ambition globale, aux Rapports Kinsey. Le projet peut être considéré comme l'une des retombées du Mouvement de Mai 68 et du développement de l'utilisation de masse de la contraception orale. En revendiquant le droit au plaisir, il se situe dans la lignée de Kinsey comme l'expression d'un certain optimisme sexuel (Béjin: 1982). Par contre, il développe une conception de la sexualité qui articule le comportement sexuel avec des systèmes d'attitudes et de représentations idéologiques; il valorise très fortement la dimension de l'amour et du couple et la maîtrise de la procréation. En outre, le rapport Simon met en oeuvre des méthodes d'investigation différentes de celles de Kinsey. II utilise un questionnaire standardisé qui est soumis à un échantillon représentatif de la population française constitué par la méthode des quotas. II fait appel à des

enquêteurs professionnels spécialisés dans les sondages d'opinion. Comme les 
Rapports Kinsey, le Rapport Simon a constitué un événement médiatique, culturel et politique, autant ou sinon plus qu'un événement scientifique.

Simon propose d'emblée, une définition étendue du comportement sexuel qui prend en compte les dimensions comportementales, relationnelles, affectives et culturelles:

"En matière de comportement sexuel, le phénomène c'est aussi bien la réaction biochimique élémentaire du neurone que l'action créatrice la plus élaborée. Les interactions, les relations réciproques et la complexité des relations humaines et donc des aspects affectifs et sexuels de ces relations rendent difficile l'analyse du comportement sexuel" (p.14)..."la comptabilité des orgasmes, masturbations, rapports homosexuels est incompatible avec la préhension de la vie vraie sexuelle de l'individu: c'est l'ambition de notre Rapport de s'efforcer de situer le comportement sexuel en fonction des attitudes et de l'information reçue. Les normes de la sexualité occidentale dans ses structures actuelles sont mal connues. Notre Rapport contribue à leur approche scientifique et facilitera leur critique." (Simon: 1972, p.17).

II adopte ainsi une position critique à l'égard de Kinsey, fondée sur une théorie implicite de la complexité (comme l'on dirait aujourd'hui) de la sexualité humaine qui rendrait celle-ci "incompatible" avec la "comptabilité". Cette complexité l'amène à élargir l'objet de sa recherche: les attitudes, les opinions, l'information et les normes concernant la sexualité sont partie intégrante du champ de la sexualité au même titre que le comportement qui en serait une résultante. Abordant le "premier rapport sexuel", il explicite l'objet de son travail:

"Dans l'enquête, nous avons étudié "le rapport sexuel complet". Si la question posée précise qu'il s'agit d'un rapport sexuel complet, c'est pour qu'il soit bien clair que l'acte sexuel s'est traduit par une intromission de la verge dans le vagin. Que le rapport ait entrainé une éjaculation ou pas, que celle-ci se soit faite à l'intérieur ou à l'extérieur du vagin, que le rapport sexuel ait été suivi d'un orgasme de la partenaire ou pas, quelle que soit la position utilisée au cours du rapport, nous étudions le rapport sexuel complet par opposition au flirt caractérisé par les caresses, attouchements et baisers. (....) De la "pratique sexuelle" d'un individu se dégage une expérience sexuelle qui ne se limite pas aux "pratiques sexuelles" dont l'histoire éclaire toute la vie affective et sexuelle de la personne." (Simon: 1972, p.198).

Le coït hétérosexuel génital constitue donc le centre de la conception du comportement sexuel. Celui-ci sera donc étudié, tout d'abord, du point de vue de ses différentes dimensions techniques (certaines positions coïtales adoptées) dont une liste est proposée dans le questionnaire, et qui fera l'objet d'une analyse détaillée (notamment en fonction des facteurs retenus par Simon: l'âge, le niveau d'études, I'habitat et la pratique religieuse). Les exemples choisis pour se démarquer de Kinsey (orgasmes, masturbations, rapports homosexuels) sont illustrés par les composantes des pratiques sexuelles qui seront reléguées en second plan par Simon dans son abord de "la vie vraie sexuelle d'un individu ".

La conception du comportement sexuel, selon Simon, est construite selon un double mouvement. D'une part, on est confronté à une intégration dans un champ de 
la sexualité défini extensivement par la prise en compte de dimensions psychoaffectives et sociologiques. D'autre part, à une centration sur un type spécifique d'activité sexuelle. Le terme de comportement sexuel qui sert à désigner l'ensemble n'est pas retenu pour désigner les activités spécifiques à proprement parler; c'est le terme de "rapport sexuel complet" qui désignera le versant "comportemental" de l'étude.

\section{les aspects de l'activité sexuelle}

L'étude détaillée du questionnaire et de la présentation des résultats nous permet de comprendre comment les prémisses théoriques et l'idéologie que nous venons de présenter ont été opérationnalisées dans le cadre d'un projet qui vise à "étudier la vie sexuelle des Français sous le plus grand nombre d'aspects significatifs." (p.138).

Le questionnaire est structuré selon trois axes: la formation et l'expérience, les attitudes à l'égard de la sexualité, les comportements sexuels qui présideront à l'organisation des résultats et au calcul des corrélations visant à la construction des typologies.

L'axe "formation - information et expérience acquises" attribue, comme prévu, un statut dominant aux "rapports sexuels complets" et hétérosexuels qui apparaissent comme le pivot de la vie sexuelle et de sa formation, autour desquels les autres aspects sont organisés au titre d'expériences secondaires. Ces "autres expériences" regroupent la masturbation, les pollutions nocturnes, l'homosexualité et les maladies vénériennes. La vie sexuelle ainsi définie est replacée dans le contexte social conjugal ou extra-conjugal. Les techniques sexuelles (positions et situations) sont traitées de manière détaillée et prises en compte au niveau de l'analyse (ce qui constitue une nouveauté par rapport à Kinsey). Le Rapport accorde une large place à la dimension des pratiques effectives et à leurs différents aspects. Par contre, on est surpris de constater que la sexualité infantile n'est abordée dans le Rapport Simon que sous l'angle de l'éducation sexuelle et de l'influence du milieu familial; de même pour la vie sexuelle des adolescents. La vie sexuelle semble débuter à l'occasion du "premier rapport sexuel complet". Contrairement à Kinsey, Simon ne propose pas d'anamnèse de la vie sexuelle qui remonterait aux premières activités sexuelles. Simon limite l'investigation à certains aspects de la vie sexuelle adulte du point de vue de l'hétérosexualité. Dans un autre domaine, l'attention est focalisée sur les informations anatomo-physiologiques et la connaissance technique et pratique des méthodes contraceptives. Une série de questions sont consacrées à l'évaluation de la notoriété de l'institution et des services du Planning Familial. 
La deuxième partie, consacrée au "déroulement habituel des rapports sexuels", précise et renforce la centration sur le coït hétérosexuel. Les autres techniques sexuelles (caresses, contacts bucco-génitaux et contacts anaux) apparaissent au titre de compléments ou de variantes de substitut éventuel. Le coït est traité sous l'angle de sa dimension quantitative (n'est-ce pas une comptabilité que l'on voit à l'oeuvre ?), et du point de vue de l'appréciation de la satisfaction individuelle et relationnelle qu'il procure.

La troisième partie traite des attitudes à l'égard de l'institution matrimoniale et de son évolution, de l'éducation sexuelle des jeunes, de l'aspiration à une meilleure information en matière sexuelle, de la contraception et de l'avortement, de la prostitution et de l'érotisme. Une section est consacrée au rôle de la sexualité par rapport au bonheur et la santé.

Cet ensemble témoigne d'une approche sélective orientée par une préoccupation majeure concernant le développement de la contraception moderne décrite comme l'entrée dans "la civilisation contraceptive ". Cette conception a plusieurs conséquences. D'une part, elle implique une focalisation sur le coït hétérosexuel parce que celui-ci est le lieu d'exercice potentiel et effectif de la contraception. Cette focalisation se fait au détriment d'autres aspects de l'activité sexuelle qui ne sont pas (ou peu) pris en compte. Elle transparaît très nettement dans la construction du questionnaire qui accorde une place centrale aux "expériences hétérosexuelles" abordées du point de vue du "déroulement habituel des rapports sexuels". La lecture du questionnaire montre que Simon n'a pas rompu avec la "comptabilité des orgasmes" mais que celle-ci est limitée aux "rapports sexuels complets" avec un partenaire de sexe opposé. La masturbation, les pollutions nocturnes et les rapports avec une personne du même sexe n'émargent qu'en fin de questionnaire dans la rubrique "Autres expériences sexuelles" au même titre que "les M.S.T., l'expérience de la nudité des parents, les perceptions de la sexualité des parents et ... l'expérience de la passation du questionnaire". Par contre, la violence sexuelle ou la violence comme dimension des actes sexuels est totalement passée sous silence aussi bien en ce qui concerne les rapports sexuels que les attitudes et opinions.

La focalisation sur la procréation et la contraception a imposé un biais de méconnaissance dans le sens d'une réduction normative des comportements sexuels aux rapports hétérosexuels. La structure de ce questionnaire apparaît donc en relatif décalage avec les intentions déclarées.

\section{les typologies}

L'apport le plus original du Rapport Simon consiste en la construction des typologies. Cette construction a été effectuée en deux étapes. D'une part, les 
typologies fondées sur chacune des trois grandes catégories du questionnaire: type de formation, attitudes par rapport à la sexualité, et comportements sexuels, construites à partir des résultats obtenus par chacun des sexes. D'autre part, les typologies globales fondées sur le croisement des trois typologies spécifiques. Cette analyse typologique en deux étapes aboutit aux "types" suivants.

Pour les hommes, on distingue: "les vierges"; "les pudiques"; "les peu informés, peu expérimentés, de formation stricte"; "les mieux informés" et "les plus expérimentés". Pour les femmes: "les vierges"; "les pudiques"; "les vertueuses ignorantes; "les vertueuses informées"; "les mieux informées".

Les regroupements opérés précisent l'orientation et le message contenus dans le Rapport Simon. II s'agit de mesurer les changements intervenus dans la vie sexuelle de la population française sous l'effet de la contraception et de l'information qui l'accompagne. L'extension qui est donnée à la définition de la vie sexuelle fait quasiment de celle-ci un indicateur des modes de socialisation, de l'accès à la culture scientifique, de la gestion des relations interpersonnelles et de la morale pratique des individus et de certaines de ses composantes psychologiques. En d'autres termes, les analyses typologiques mettent en évidence des corrélations élevées entre la connaissance de l'information scientifique, les attitudes considérées comme "évoluées", c'est à dire faites d'intérêt et de tolérance à l'égard de certaines pratiques (avortement; contraception; rapports pré-nuptiaux; relations extra-conjugales) ${ }^{9}$, la disposition à parler des problèmes affectifs et sexuels, et la satisfaction obtenue ou espérée de l'activité sexuelle. Cette typologie est assez semblable pour les hommes et les femmes.

"les mieux informés: (....) Deux profils assez différents se rencontrent parmi eux: les uns, jeunes et célibataires, ont fréquemment parlé de problèmes sexuels avec leur entourage, ont lu des livres d'éducation sexuelle, se déclarent bien informés sur les moyens de contraception. Ils sont satisfaits de leur vie sexuelle tout en déclarant qu'ils aimeraient bien avoir plus de rapports. Les autres, mariés et entre deux âges, ont des caractéristiques semblables aux précédents mais moins marquées. (Simon: 1972, p.428)

Par contre, Simon n'attribue pas la même signification à la quantité d'activité sexuelle selon qu'il s'agit des hommes ou des femmes. Pour les hommes, l'indicateur de la quantité n'est pas corrélé de manière positive avec l'information scientifique et la morale qui l'accompagne:

"les plus expérimentés : Ce type se caractérise par la précocité de l'activité sexuelle des individus qui le composent et leur expérience acquise. (....) Les hommes de ce groupe sont en grande majorité âgés de plus de trente cinq ans et les opinions qu'ils

9 On est surpris de constater que les attitudes à l'égard de l'homosexualité ne sont pas abordées dans ce Rapport. Par contre, l'enquête "Sex and Morality in the U.S." (Klassen et coll. 1989) réalisée aux Etats-Unis à la même époque et commandée par le N.I.M.H. (National Institute of Mental Health) est fondée sur l'évaluation des attitudes et des mentalités à l'égard de l'homosexualité. 
expriment sur les questions faisant appel aux connaissances trahissent leur manque réel d'information scientifique et objective. Dans ce groupe, se rencontrent plus particulièrement: des hommes ayant eu leur premier rapport hétérosexuel très jeunes et ayant connu un grand nombre de prostituées; des hommes de niveau d'études primaire, ayant peu lu de livres d'éducation sexuelle et mal informés sur les moyens contraceptifs actuels. (....) A la différence des "mieux informés", les "plus expérimentés" apparaissent fréquemment comme des "égoïstes expérimentés". (Simon: 1972, p.429, et 519).

Pour les femmes, l'information et l'accès à la contraception sont présentés

comme ayant un effet libérateur et favorisant l'épanouissement sexuel.

"les mieux informées : Ce type se différencie nettement des quatre autres. Les femmes qui le composent manifestent un niveau de connaissances et d'expérience élevé. Leur premier rapport a eu lieu assez tôt, il a été précédé de flirt; elles ont eu en moyenne plus de partenaires que les autres femmes. Elles s'estiment très ou assez bien informées des moyens contraceptifs et des problèmes de l'éducation sexuelle des enfants; beaucoup ont lu des livres d'éducation sexuelle ou érotiques. Elles émettent sur la sexualité des opinions plus souvent pertinentes, aussi bien sur le rôle de la dimension du sexe masculin dans la satisfaction de la femme pendant l'acte que sur les capacités orgastiques de l'homme et de la femme ou sur la nature des causes de l'impuissance ou de la frigidité." (Simon: 1972, p.441).

"La formation apparaît ici, à l'évidence, comme un facteur déterminant de l'épanouissement sexuel de la femme." (Simon: 1972, p. 524).

\section{le champ du sexuel et du non-sexuel}

On assiste à un renversement de perspective par rapport à Kinsey. Pour ce dernier, les facteurs non-sexuels permettaient d'expliquer la fréquence et le choix des sources de l'activité sexuelle. Pour Simon, c'est l'activité sexuelle, sa mesure et son analyse qui constitue le facteur permettant de dégager le sens de la sexualité et de comprendre le fonctionnement de l'idéologie et de la vie affective des individus.

Par ailleurs, Simon se situe dans une perspective de changement des mentalités et des pratiques (notamment en ce qui concerne la contraception). II met en évidence que la diffusion de la connaissance rationnelle et scientifique contribue à la modification de ces pratiques et qu'elle est corrélée avec un changement de mentalités sur le plan des relations inter-individuelles.

La sexualité apparaît comme un élément central de la culture et de la société. Deux dimensions sont particulièrement privilégiées: la procréation et la santé mentale. Le contrôle de la procréation à l'aide de la contraception doit favoriser un exercice harmonieux de la sexualité dégagé de ses peurs et culpabilités archaïques, lequel doit à son tour participer de l'équilibre psychique des citoyens.

"Une sexualité bien entendue, un équilibre psycho et socio-affectif demeurent dans une civilisation technicienne, le seul bouclier préservant des troubles psychiques et de l'insécurité morale." (Simon: 1972, p.23). 
La connaissance rationnelle et scientifique de la sexualité et sa diffusion par le biais de l'éducation sexuelle sont les moyens de remplir ce programme. La valorisation positive de la sexualité (d'une sexualité "bien entendue") est présentée comme un élément déterminant du fonctionnement harmonieux de l'individu dans le champ social. La réalisation de l'enquête est présentée comme un élément du dispositif devant amener à une modification des représentations concernant le rôle de la sexualité dans l'existence:

"La nature des problèmes abordés dans ce questionnaire et la progression des questions posées devaient en effet amener les interviewés à prendre conscience des implications nombreuses et importantes de la sexualité sur le plan social, familial et individuel, valoriser par là le but de l'enquête et contribuer à réduire les résistances conventionnelles à faire état de son expérience intime en ce domaine." (Simon: 1974, p.139).

LES RECHERCHES ACTUELLES (Action Concertée C.E., NORC, O.M.S.). La sexualité comme vecteur de transmission d'une infection.

Ces recherches sont suscitées par une forte demande sociale qui se situe dans le contexte du phénomène du sida et qui est à l'origine des sollicitations institutionnelles (publiques, nationales et internationales), et de l'engagement de moyens financiers d'une ampleur jamais atteinte en ce domaine. On remarque cependant que dans de nombreux pays, notamment aux Etats-Unis et au RoyaumeUni, l'engagement des fonds publics dans ces recherches a été suspendu ou retardé pour des raisons politiques et idéologiques. Les recherches dans le domaine de la sexualité restent marquées d'un tabou que la dramatisation qui accompagne le phénomène du sida ne parvient pas toujours à lever; comme si "penser la sexualité au niveau de ce que font les corps, est le commencement de la réalisation d'un acte sexuel - un acte avec ses propres normes et contraintes, et en même temps, un acte qui provoque les réactions physiologiques de ce que Masters et Johnson ont désigné comme l'excitation sexuelle." (Gagnon, Simon: 1973, p.5) .

Les documents qui font l'objet de la discussion sont des projets émanant de groupes leaders dans la recherche en ce domaine tant sur le plan national (NORC aux Etats-Unis) qu'international (O.M.S., A.C.C.E.). La position stratégique occupée par ces différents groupes les met en situation de définir les grandes orientations et les problématiques de recherche pour les années à venir. Cette situation constitue une nouveauté importante dans le champ des recherches sur la sexualité, dans la mesure où se met en place une tentative de structuration d'un milieu de recherche à un niveau quasi-industriel, disposant (ou pouvant disposer) d'importantes sources de financement. Si tous ces projets sont explicitement construits en référence à la problématique du sida (laquelle justifie leur existence et la mise à disposition de 
financements), ils proposent des constructions différentes des relations entre sida, sexualité et comportement sexuel et prennent en compte des variables sociologiques et/ou psychologiques différentes. Malgré leurs différences, ces projets sont tous reliés organiquement. Certains des auteurs ont participé à la préparation de plus de l'un de ces projets. Du point de vue de notre questionnement sur le devenir de la recherche empirique en sciences sociales dans le champ de la sexualité, il importe d'examiner les conséquences d'une situation dans laquelle "la sexualité est traitée dans la perspective du sida plutôt que le sida dans la perspective de la sexualité " (Gagnon: 1988, p.593).

Le recours au concept de comportement sexuel est lié à la découverte du rôle de certaines pratiques sexuelles dans la transmission du VIH. L'hypothèse dite du "style de vie" a été formulée lors de l'apparition des premiers cas de pneumocystose chez quelques hommes qui présentaient la caractéristique commune d'avoir des relations sexuelles avec un nombre élévé de partenaires du même sexe, des pratiques anales, et parfois, d'inhaler du nitrite d'amyle (Oppenheimer: 1988). Partant de là, il s'est avéré nécessaire de mesurer l'incidence de ces pratiques (et des pratiques qui ont été progressivement définies "à risque") en tenant compte des caractéristiques sociales des individus et de leurs réseaux de socialisation afin de définir les risques d'exposition à l'infection. C'est dans ce contexte que la connaissance de certaines pratiques effectives et de leur incidence au sein de certains groupes ainsi que dans la population dite générale est apparue de première nécessité et trouve une justification scientifique et sociale.

"En référence à la maladie, l'incidence, la régularité et la prévalence des comportements sexuels fournit les paramètres physiques de base de la transmission, alors que les contextes et les liens sociaux donnent accès aux paramètres sociaux de la diffusion." (Laumann et coll., p.8).

En d'autres termes, il s'est avéré nécessaire, d'un point de vue épidémiologique, de savoir ce que les individus font effectivement - leurs comportements - et avec quels partenaires, pour évaluer le nombre et les caractéristiques des personnes infectées effectivement ou potentiellement. II s'agit bien d'une connaissance située à un niveau biologique. C'est dans une optique de prévention, que la connaissance des "déterminants sociologiques et psychologiques" de ces comportements est considérée comme pertinente pour tenter de modifier les "comportements à risque". Partant de là, on a réalisé de surcroît, que l'on manquait d'informations fiables et récentes sur les comportements sexuels de la population générale. Cet ensemble a favorisé la mise en oeuvre de grandes enquêtes sur le comportement sexuel de la population générale.

Cette problématique de recherche pose trois questions: quelles sont les définitions données au comportement sexuel et les pratiques qui le constituent? Quel est le statut théorique des "déterminants" ? Quels sont les statuts respectifs et les 
modèles d'articulation établis entre le niveau des comportements et le niveau des "déterminants" ?

\section{Les définitions du comportement sexuel}

Le "comportement sexuel" apparaît de manière assez variable dans les documents étudiés. II apparaît dans les titres généraux des recherches ou bien il sert à en désigner l'objet central. Son utilisation variable et non systématique donne l'impression d'un embarras des chercheurs. Comme s'il était nécessaire d'employer ce terme tout en sachant bien qu'il est relativement inadéquat pour traiter de l'objet de la recherche.

Dans le texte intitule "Life Course and Network Considerations in the Design of the Survey of Health and Sexual Behavior" ("Considérations sur I'histoire de vie et les réseaux dans le projet d'une enquête sur le comportement sexuel et de santé") les auteurs emploient les termes de "pratiques sexuelles", "conduites sexuelles", "activité sexuelle", "contact sexuel", "transaction sexuelle", "vie sexuelle", "script sexuel" pour tenter de définir l'objet de la recherche avant de s'arrêter sur une définition opérationnelle de la sexualité entendue comme activité sexuelle:

"Au lieu de rapport, intromission ou orgasme, nous avons choisi de donner une définition plus large à l'activité sexuelle: toute activité volontaire et mutuelle avec une autre personne qui implique un contact physique et une excitation sexuelle (c'est à dire se sentir vraiment excité) même si le rapport ne s'est pas produit." (NORC: 1989, p.18).

Cette définition est relativement large dans la mesure où elle ne limite pas le champ de l'activité sexuelle à certaines sources corporelles ni à une orientation sexuelle plutôt qu'à une autre. Elle en limite cependant la portée, à l'activité sociosexuelle, en excluant implicitement les situations de contrainte (où l'activité pourrait ne pas être volontaire) et la masturbation. Cette définition apparaît indépendante de la problématique du sida qui n'est pas intégrée d'entrée de jeu à la définition de l'activité sexuelle.

Le projet O.M.S. intitulé "Relations entre partenaires et le risque d'infection à VIH" adopte un autre point de vue:

"Le comportement sexuel: au sens le plus large [il] recouvre une vaste gamme d'activités à orientation sexuelle, allant de l'amitié aux rapports homo ou hétéro sexuels. Il englobe également les méthodes de contraception, notamment l'usage de préservatifs. II est influencé par l'idéologie sexuelle, l'attitude à l'égard du risque et l'opportunité. Mais il exerce aussi en retour, une influence sur ces trois facteurs." (O.M.S. p.4)

On est confronté à une définition large du comportement sexuel pouvant englober des situations supposées sans contact corporel. Cependant, d'entrée de jeu, la définition introduit "la contraception" et "l'usage de préservatifs" comme éléments 
constitutifs du comportement, et "l'attitude à l'égard du risque" comme facteur déterminant les comportements.

Le projet A.C.C.E. se situe de manière plus spécifique dans la problématique du sida. On ne trouve pas de définition générale du comportement sexuel pouvant fonctionner comme cadre de référence. D'emblée, les comportements sexuels sont construits et classés en référence au risque d'infection par le $\mathrm{VIH}$.

"Quelle est la prévalence des comportements sexuels associés, positivement ou négativement, avec le risque d'infection au VIH ? (...) L'objectif principal est de chercher le degré d'exposition des individus au risque de contamination par le $\mathrm{VIH}$ du fait de leurs pratiques sexuelles. Etant donnés les nombreux aspects du comportement sexuel, nous devons utiliser des critères rigoureux pour choisir les variables nécessaires à l'opérationnalisation du concept d'exposition au risque. Si nous étions intéressés par un autre problème que le risque d'infection au $\mathrm{VIH}$, d'autres aspects du comportement sexuel devraient être pris en considération." (A.C.C.E.: 1989, p.3).

Ces critères sont les suivants: "fréquence de la sexualité ("sex" dans le texte anglais) protégée et non-protégée; "position dans les réseaux des échanges sexuels"; "occasions pour les échanges sexuels"; "modèles de relations".

Si la recherche est focalisée, à un premier niveau, sur les comportements considérés du point de vue du risque d'infection par le $\mathrm{VIH}$, par contre au niveau de l'appréciation des changements de comportements une attention particulière est accordée à "la recherche des nouveaux plaisirs qui semblent se situer de plus en plus à un niveau fantasmatique (minitel, peepshows, etc...)." (A.C.C.E., p.7).

Le terme de comportement sexuel est utilisé, dans ce dernier projet, dans deux sens différents: il renvoie à fois à une acception générique, et à des pratiques spécifiques définies selon des critères rigoureux, ce qui en excluerait d'autres du fait de leur non-pertinence par rapport au risque. Par ailleurs, il est envisagé de prendre en compte des "pratiques nouvelles de recherche du plaisir" qui semblent étrangères à l'acception habituelle du champ du "comportement sexuel". Une fois de plus, on est confronté à un schéma qui se situant dans un champ défini implicitement comme celui du "comportement sexuel", propose de traiter certains des aspects de celui-ci et des pratiques qui jusque là étaient considérées comme extérieures à son champ.

L'analyse de ces définitions laisse entrevoir l'importance de l'influence de la problématique du sida sur la définition du champ du "comportement sexuel".

\section{les aspects du comportement sexuel}

La lecture comparée du protocole général (O.M.S.), et de la présentation du questionnaire fait apparaître des décalages entre un certain nombre de points. Comme nous l'avons noté plus haut, le protocole de recherche donne une définition relativement large du comportement sexuel. Par contre, le document de présentation du questionnaire circonscrit le domaine d'investigation. On propose de se limiter à "la 
mesure des facteurs de risque de transmission hétérosexuelle au sein de la population adulte en prenant en compte les éléments suivants suggérés par les enquêtes séroépidémiologiques: (a) choix des partenaires sexuels; (b) nombre de partenaires sexuels; (c) utilisation de préservatifs en latex; (d) présence d'autres maladies transmissibles sexuellement; (e) type de rapports sexuels" (p.2).

Le champ ainsi défini accorde une place importante à la dimension des partenaires sexuels et à l'étude de certaines de leurs caractéristiques. L'utilisation de préservatifs en latex est associée aux pratiques contraceptives et à l'anamnèse des M.S.T. ainsi qu'à leur prévention. Tous ces points font l'objet d'une investigation beaucoup plus détaillée que dans les Rapports Kinsey et Simon. Concernant le "comportement hétérosexuel" le document constate la difficulté de l'investigation et, arguant "de la fatigue, des défaillances de mémoire et des réticences culturelles qui font hésiter à parler de son comportement sexuel ", propose de limiter le questionnement rétrospectif sur une durée limitée à une année. A ce stade du document le comportement sexuel ou hétérosexuel n'est pas défini. Le projet NORC propose, pour sa part, de le faire remonter à cinq ans pour tenter d'évaluer les changements intervenus sous l'effet du sida.

Compte-tenu des difficultés envisageables, le document OMS prévoit des sections facultatives portant sur "l'usage de la drogue " (désigné deux lignes plus loin comme "abus de drogues "), "certaines pratiques sexuelles particulières telles que les pratiques anales " et les "injections ". La présence de ces items est justifiée "en raison de leurs relations établies avec le risque de transmission du VIH ".

Ce point pose problème. En effet si le but de cette enquête est "la mesure des facteurs de risque de transmission hétérosexuelle au sein de la population adulte ", il est surprenant de devoir justifier la présence dans le questionnaire, de questions renvoyant directement à des pratiques connues pour leur rôle dans la transmission du VIH et désignées comme "particulières". II est d'autant plus surprenant d'en faire l'objet de questions facultatives laissées à l'initiative de "l'enquêteur principal sur chaque site d'enquête ". Les auteurs du document recommandent cependant vivement leur intégration dans le cadre du questionnaire.

Laumann et coll. présentent une construction plus systématique:

"Les travaux épidémiologiques actuels suggèrent que l'efficacité de la transmission de la maladie varie en fonction de la technique sexuelle employée. Il est donc nécessaire de recueillir des informations spécifiées en fonction de chaque partenaire sur au moins trois techniques: la fréquence des rapports anaux (en distinguant l'introducteur et le récepteur), des rapports oraux et des rapports vaginaux, pour chaque partenaire, dans chaque séquence temporelle." (Laumann et coll. p. 7).

II apparaît donc clairement que le fait de se situer explicitement dans le contexte du sida ne constitue pas une raison suffisante pour aborder les différents aspects du comportement sexuel et notamment ceux auxquels on attribue un statut particulier. La reconstruction du "comportement sexuel" autour de trois types de 
contact retenus du fait de leur efficacité relative par rapport à la transmission du VIH reste encore dépendante, dans certains cas, des idéologies sexuelles des chercheurs et de contraintes idéologiques ou politiques.

Le thème de "la drogue " pose un autre type de question. D'une part, on ne spécifie pas la nature des produits introduits dans l'organisme ni leur mode d'introduction, alors que l'on sait que c'est l'échange de seringues qui est l'un des vecteurs de la transmission du VIH, indépendamment du produit injecté. D'autre part, on tient pour acquise la justification du fait qu'on insère la question de "la drogue" dans un protocole de recherche sur les comportements sexuels. Ce thème est-il inséré dans le questionnaire du fait de la dimension sexuelle de la drogue ou du fait du rôle de certaines pratiques considérées comme non-sexuelles dans la transmission du virus? La focalisation sur "la drogue" laisse dans l'ombre des pratiques pouvant avoir une effectivité dans la transmission du virus telles que les tatouages, les scarifications ou les saignées lorsque elles sont réalisées dans des conditions d'hygiène non conformes aux règles définies.

Enfin, alors que le protocole et le document de présentation du questionnaire se situent dans le champ du "comportement hétérosexuel" on est surpris de lire dans le questionnaire "Partner Relations Survey Phase I Men" (12 Janvier 1989) un ensemble de questions ayant trait à des pratiques avec des partenaires du même sexe que le répondant. Le questionnaire se donne les moyens de prendre en compte les relations avec un partenaire du même sexe aussi bien qu'avec un partenaire de sexe différent. La notion de "partenaire régulier" a un double objectif; elle permet de désigner le (ou les) "partenaire régulier" éventuel de même sexe que le répondant en même temps que celui (ou ceux) de sexe différent (en y incluant "l'épouse"). Il propose d'englober cet ensemble de choix de partenaire dans une échelle à 5 points inspirée de l'échelle "H-H" à 7 points de Kinsey. Par ailleurs, les versions masculine et féminine du questionnaire n'accordent pas la même attention aux pratiques homosexuelles. Les femmes ne sont pas interrogées sur ce thème dans le détail de leurs pratiques.

Sur ce point précis (le choix du sexe (gender) du partenaire) le questionnaire apporte un éclairage spécifique sur ce qu'on désigne actuellement sans plus de définition comme "bisexualité". En ce sens, il répond à certaines des données épidémiologiques concernant l'importance de la transmission du virus lors de certaines pratiques et à la propagation de celui-ci en dehors des cercles pratiquant strictement des relations avec des partenaires de même sexe. Mais, ce faisant, il se situe en décalage par rapport aux intentions énoncées dans le préambule, qui visaient à l'étude de la "transmission hétérosexuelle". Ainsi la description du comportement hétérosexuel adulte, supposée constituer l'objectif principal du questionnaire, est-elle englobée dans 
un cadre opérationnel qui remet en question les distinctions théoriques strictes entre comportement hétérosexuel et homosexuel (cf. Gagnon: 1990).

On constate ainsi que la liaison entre le comportement sexuel et le sida produit les effets suivants sur la définition du champ de ce comportement. D'une part, on retient certains comportements considérés comme sexuels et on en exclut d'autres du fait de leur non relation immédiate avec la transmission du VIH; d'autre part, on prend en compte certaines pratiques considérées comme non-sexuelles mais ayant un rapport avec la transmission du VIH, mais pas l'ensemble de ces pratiques. On peut supposer qu'une reconstruction du "comportement sexuel" se met en place.

\section{les déterminants et les contextes du comportement sexuel.}

La problématique du sida fait ressurgir la problématique classique de l'influence psycho-sociale de la connaissance scientifique sur les comportements, tant sur le plan vertical (diffusion de messages sociaux) qu'horizontal (influence des réseaux d'appartenance sociale et de relations interpersonnelles, selon le modèle Lewinien). Les références à la contraception constituent un modèle de changement des pratiques sous l'effet du processus de rationalisation (Béjin, Pollak: 1977). C'est ce modèle qui est exporté dans l'étude de l'utilisation des préservatifs et l'adoption des pratiques à moindre risque.

En outre, le phénomène du sida fait apparaître des problématiques nouvelles dans le champ de la sexualité: les réseaux de partenaires sexuels et la notion de risque, qui constituent la marque d'une évolution des constructions sociales de la sexualité.

La problématique des réseaux témoigne d'une double évolution de la pensée. D'une part, l'approche de la sexualité est focalisée sur la dimension relationnelle considérée comme principe organisateur des pratiques sexuelles et de leur distribution chez un même sujet (ce qui nous renvoie aux prémisses posées par Burgess). D'autre part, elle entérine le phénomène culturel de la reconnaissance du multipartenariat sexuel (simultané et séquentiel) comme réalité sociale. "Plus de la moitié des adultes ont l'expérience d'au moins un changement de partenaire principal au cours de leur vie." (NORC: 1987, p.23). La prise en compte du multipartenariat constitue une évolution par rapport à la norme de la conjugalité qui constituait le "partenaire régulier" (souvent le conjoint) comme partenaire central. Dans le projet NORC, le "partenaire régulier" ou principal se trouve inscrit dans une double perspective. Sa position est relativisée par rapport à d'autres partenaires effectifs ou potentiels dans la même séquence temporelle. L'ensemble de ces partenaires est considéré théoriquement (dans le modèle) comme pouvant être aussi bien du même sexe que d'un sexe différent du répondant. Ce qui témoigne de l'émergence de "la bisexualité". En outre, le "partenaire régulier" est traité dans dans la dynamique socio- 
temporelle de l'individu avec des séquences de présence ou d'absence de ce partenaire, des séquences de rupture et de renouvellement. La configuration du réseau de chaque individu ainsi que son évolution sont considérés comme des facteurs déterminants des types de pratiques sexuelles mis en acte ainsi que de l'attitude face au risque.(NORC, 1987).

La problématique du risque est importée d'une part, de champs hétérogènes au domaine de la santé (Douglas, Calvez : 1990) ainsi qu'au domaine du rôle du tabac dans la genése des cancers (Brandt: 1990). La notion de risque était employée de manière ponctuelle pour l'analyse des MST et des grossesses non-désirées. Le champ de la sexualité n'avait pas encore été traité globalement du point de vue du risque. Cette problématique est en voie de construction dans la mesure où l'on a pu assister au cours des dernières années à des déplacements de formulation. Depuis, les groupes à risques, les sujets à risques, les partenaires à risque, les comportements à risque et les attitudes face au risque, la notion de risque oscille entre des acceptions qui la situent dans une certaine objectivité ou comme une dimension d'un processus psycho-social. Le problème est bien trop complexe pour être discuté rapidement sur le fond. Notons simplement, qu'il s'agit d'une catégorie nouvelle qui produit des effets sur la construction du comportement sexuel d'une part, en focalisant l'attention sur certaines formes de contact au détriment d'autres formes ou des modalités de ces contacts (par exemple l'analyse des différentes positions coïtales n'est plus prise en compte) et d'autre part, en y intégrant des pratiques pouvant difficilement être considérées comme faisant partie du comportement sexuel telles que l'injection de toxiques par voie intraveineuse. En outre, la thématique du risque introduit la distinction entre pratiques "protégées" et "non-protégées" en fonction de l'utilisation de préservatifs ainsi que le concept de "sexualité à moindre risque". Enfin, cette classification introduit la prise en compte de modes de satisfaction dits "substitutifs" et notamment de produits offerts (ou plutôt vendus) par l'industrie du sexe (cf. Faligot, Kauffer: 1987).

L'introduction de la notion de risque dans le champ du comportement sexuel laisse, à son tour entrevoir les prémisses d'un redécoupage idéologique de l'activité sexuelle en fonction de la relation avec le risque de contamination. En d'autres termes, le risque semble être en passe de devenir l'un des critères permettant de déclarer qu'une pratique sexuelle est "normale" ou pas. Ce redécoupage ne s'effectue bien évidemment pas sur un terrain vierge. II se surajoute aux classifications déjà existantes et empruntées à d'autres registres: moral, juridique, psychanalytique. 
L'utilisation du concept de comportement sexuel n'est donc pas sans poser de problèmes quant à la définition du champ et des activités qu'il recouvre et qu'il vise à traiter. Ce concept est l'objet d'une contradiction centrale. Relevant du champ de la biologie et d'approches naturalistes de la sexualité humaine, il est surprenant de constater que son exportation dans le champ des sciences sociales lui apporte une flexibilité qui est soumise aux aléas des différents contextes sociaux, culturels et idéologiques qui suscitent son utilisation. Le concept est soumis à des variations importantes sur le plan des activités, à proprement parler; nous avons pu montrer que des activités retenues par Kinsey ne le sont plus dans les autres recherches, et inversement. C'est surtout le critère de définition du "sexuel" qui reste problématique. Alors que chez Kinsey l'utilisation du critère de l'orgasme a fait preuve de limites et de contradictions qui sont plus ou moins habilement contournées, on constate que d'autres critères plus ou moins extérieurs et plus ou moins associés à une hypothètique définition du sexuel sont mis en oeuvre depuis Simon, pour délimiter empiriquement le champ du "comportement sexuel" et que l'utilisation de ces critères tend à remodeler, à chaque fois, la sphère du "comportement sexuel" selon des processus que nous venons de présenter. Enfin, l'utilisation de ce concept impose des statuts différents aux dimensions sociologiques et psychologiques en tant que parties prenantes ou comme déterminants.

\section{Références bibliographiques:}

Béjin A. (1982): "Crépuscule des psychanalystes, matin des sexologues", Communications , 35 , p. $159-177$.

Béjin A., Pollak M. (1977): "La rationalisation de la sexualité", Cahiers Internationaux de Sociologie, vol. LXII, pp.105-125.

Brandt A. (1990): "The cigarette, risk, and American culture", Daedalus , 119 (4), pp. 155-176).

Burgess E.W. (1949). "The sociologic theory of psychosexual behavior", in: P. H. Hock, J. Zubin, eds. Psychosexual developments in health and disease, New York, Grune and Stratton.

Caplan P. (ed.): (1987). The cultural construction of sexuality. London, Routledge.

Douglas M., Calvez M. (1990): "The self as risk taker: a cultural theory of contagion in relation to AIDS", The Sociological Review , 38, pp. 445-465.

Ellis H. (1936): Studies in the Psychology of Sex. New York

European Communities Concerted Action on sexual behaviour and risks of HIV infection (1989). Bruxelles.

Faligot R., Kauffer R. (1987): Porno Business . Paris, Fayard. 
Foucault M. (1976): Histoire de la sexualité. La volonté de savoir. Paris, Gallimard.

Gagnon J. (1988). "Sex research and sexual conduct in the era of Aids." Journal of AIDS , 1, pp. $593-601$.

Gagnon J. (1990): "Gender preferences in erotic relations: The Kinsey scale and sexual scripts" p. 177-207 in: D.P. McWhirter, S.A. Sanders, J.M. Reinisch, eds: Homosexuality/Heterosexuality: Concepts of sexual orientation. New York, Oxford University Press.

Gagnon J., Simon W. (1973). Sexual conduct. Chicago, Aldine.

Gagnon J., Simon W. (1987): "The sexual scripting of oral genital contacts" Archives of sexual behavior, 16 (1), p.3-25.

Gaignebet C. (1991): "Y a-t-il un acte sexuel pour l'anthropologie ?" Histoire des moeurs , tome 2, Paris, Gallimard,

Gebhard P. (1971): "The anthropological study of sexual behavior: definitions", in D.S. Marshall, R.C. Suggs eds. Human sexual behavior: variations in the ethnographic spectrum. New York, Basic Books.

Gebhard P. (1974): "Sexual Behaviour, Human"in: Encyclopaedia Britannica.

Kinsey A., Pomeroy W., Martin C. (1948). Le comportement sexuel de l'homme. Paris, éditions du Pavois (tr. fr.).

Kinsey A., Pomeroy W., Martin C.,Gebhard P. (1953). Le comportement sexuel de la femme . Paris, Amiot-Dumont (tr. fr.).

Klassen A., Williams C., Levitt E. (1989): Sex and morality in the U.S. An empirical enquiry under the auspices of the Kinsey Institute. Middletown, Conn. Wesleyan Univ. Press.

Krafft-Ebing R. (1965): Psychopathia Sexualis , New York.

Kuhn T. (1970). La structure des révolutions scientifiques. Paris, Flammarion (tr. fr. 1983)

Lagache D. (1948): "La conduite humaine" pp.5-8. Bulletin du groupe d'études de psychologie de l'Université de Paris. Vol.1 .

Lalande A. (1962): Vocabulaire technique et critique de la philosophie. Paris, P.U.F.

Lanteri Laura G. (1979): Lecture des perversions. Paris, Masson.

Laqueur T. (1990): Making sex. Body and gender from the Greeks to Freud. Cambridge, Harvard University Press.

Laumann E., Gagnon J., Michael R. (1989). $\quad$ "Life course and network considerations in the design of the survey of health and sexual behavior" Fifth Conference on Health Survey Research Methods. Keystone, Colorado, May 2-5 1989.

NORC. (1987): Social and behavioral aspects of health and fertility-related behavior. Technical proposal to the N.I.C.H.D.

O.M.S. (1989): "Les relations entre partenaires et le risque d'infection à VIH." protocole de recherche dactylographié. 
Oppenheimer G. (1988): "In the eye of the storm: The epidemiological construction of Aids", p.267-300 in: E. Fee, D. Fox eds. Aids, The burden of history. Berkeley, University of California Press.

Pomeroy W. (1972): Dr. Kinsey and the Institute for Sex Research. New Haven, Yale University Press.

Robinson P. (1976): The modernization of sex. New York, Harper and Row.

Signoret J.-L. (1980): "Comportement sexuel", Encyclopaedia Universalis , Vol 14. Paris.

Simon P., Gondonneau J., Mironer L., Dorlen-Rollier A.-M. (1972). Rapport sur le comportement sexuel des Français. Paris, Julliard, Charron.

Tastemain C. (1989). "Les enquêtes dans la population générale" La Recherche , 213, p.1109.

Trilling L. (1948): "Sex and science: The Kinsey Report", The Partisan Review, 15 (4), 460-476.

Turner C. (1989): "Research on sexual behaviors that transmit HIV: progress and problems." , AIDS , 3, suppl. 1, pp. S63-S69.

Zwang G. (1990): Sexologie . Paris, Masson. 